\title{
Pyélonéphrite xanthogranulomateuse bilatérale focale : à propos d'un cas
}

\author{
Souhail El Yacoubi, MD; ${ }^{*}$ Imad Ziouziou, MD; ${ }^{*}$ Mohamed Zizi, MD; ${ }^{*}$ Ahmed Jahid, MD; ${ }^{\dagger}$ Tariq Karmouni, MD; \\ Khalid El Khader, MD; ${ }^{*}$ Abdellatif Koutani, MD; ${ }^{*}$ Ahmed Iben Attya Andaloussi, MD*
}

*Service d'urologie B, CHU lbn Sina, Faculté de médecine et pharmacie de Rabat, Université Mohamed V souissi, Rabat, Maroc; † †Service d'Anatomie Pathologique, CHU Ibn Sina, Rabat

Cite as: Can Urol Assoc J 2014;8(9-10):e666-9. http://dx.doi.org/10.5489/cuaj.700

Published online September 9, 2014.

\section{Résumé}

Nous présentons l'observation clinique d'un cas rare de pyélonéphrite xanthogranulomateuse (PXG) bilatérale chez un jeune patient âgé de 17 ans. La découverte progressive de l'infection a débuté par l'apparition de lombalgies bilatérales évoluant depuis un mois, sans autre signe associé. L'urotomodensitométrie penchait en faveur de deux masses rénales bilatérales, soit une masse polaire inférieure droite et une masse polaire supérieure gauche, toutes deux de densité tissulaire. Une biopsie échoguidée a suggéré la présence d'une réaction inflammatoire non spécifique. Une néphrectomie partielle polaire inférieure droite a été effectuée devant la forte présomption de tumeurs rénales malignes. Finalement, I'histologie a orienté le diagnostic vers une PXG. Il s'agit, à notre connaissance, du sixième cas de PXG focale bilatérale rapporté dans la littérature. Notre patient a reçu un traitement antibiotique à base de fluoroquinolone pendant un mois. L'évolution clinique s'est avérée bonne, et les lésions visibles à la tomodensitométrie se sont entièrement résorbées au bout d'un mois. Le patient vit toujours après un an et présente une fonction rénale normale.

\section{Introduction}

La PXG est une forme inhabituelle de pyélonéphrite chronique bactérienne qui se caractérise par la destruction du parenchyme rénal et la formation de granulomes avec abcès, collection liquidienne et présence de cellules spumeuses ${ }^{1,2}$. Il existe 1,4 cas par tranche de 100000 cas par $\mathrm{an}^{3}$, dont la plupart sont de forme unilatérale. La PXG bilatérale est extrêmement rare. Elle met en jeu le pronostic fonctionnel rénal, et les conséquences sont souvent graves.

La PXG pose des difficultés d'ordre diagnostique qui sont notamment liées au diagnostic différentiel de tumeur rénale. Nous présentons l'observation clinique d'une PXG bilatérale chez un jeune patient âgé de 17 ans. Il s'agit, à notre connaissance, du sixième cas de PXG focale bilatérale rapporté dans la littérature.

\section{Observation}

Monsieur B. B., âgé de 17 ans, n'avait aucun antécédent pathologique personnel ni familial. Il se plaignait de lombalgies bilatérales récentes survenant depuis un mois. À l'examen clinique, ce patient était en bon état général, apyrétique et sans perte de poids. À l'examen des fosses lombaires, il y avait sensibilité, sans masse palpable.

L'échographie rénale a mis en évidence deux masses rénales échogènes, soit une masse polaire supérieure gauche et une masse polaire inférieure droite. L'urotomodensitométrie a permis de déceler deux processus lésionnels (figure 1, figure 2, figure 3) :

- I'un polaire inférieur droit mesurant $52 \mathrm{~mm} \times$ $79 \mathrm{~mm}$;

- I'autre polaire supérieur gauche mesurant $52 \mathrm{~mm}$ $\times 42 \mathrm{~mm}$.

Ces lésions étaient isodenses à l'emplacement du parenchyme rénal et faiblement rehaussées après I'injection du produit de contraste (tableau 1).

La fonction rénale et les résultats de l'examen cytobactériologique des urines étaient normaux. Le taux de leucocytes s'élevait à 8,100 éléments $/ \mathrm{mm}^{3}$. II n'y avait pas de protéinurie.

Devant la forte suspicion de tumeur rénale bilatérale, nous avons eu recours à la biopsie rénale échoguidée à l'endroit le plus accessible, soit le pôle inférieur du rein droit. Le résultat anatomopathologique pointait en faveur d'une réaction inflammatoire non spécifique faisant intervenir des cellules lymphocytes, plasmocytes et polynucléaires.

Une néphrectomie partielle droite a ensuite été réalisée. À l'analyse anatomopathologique de la pièce opératoire, on a décelé un infiltrat inflammatoire constitué d'amas d'his- 


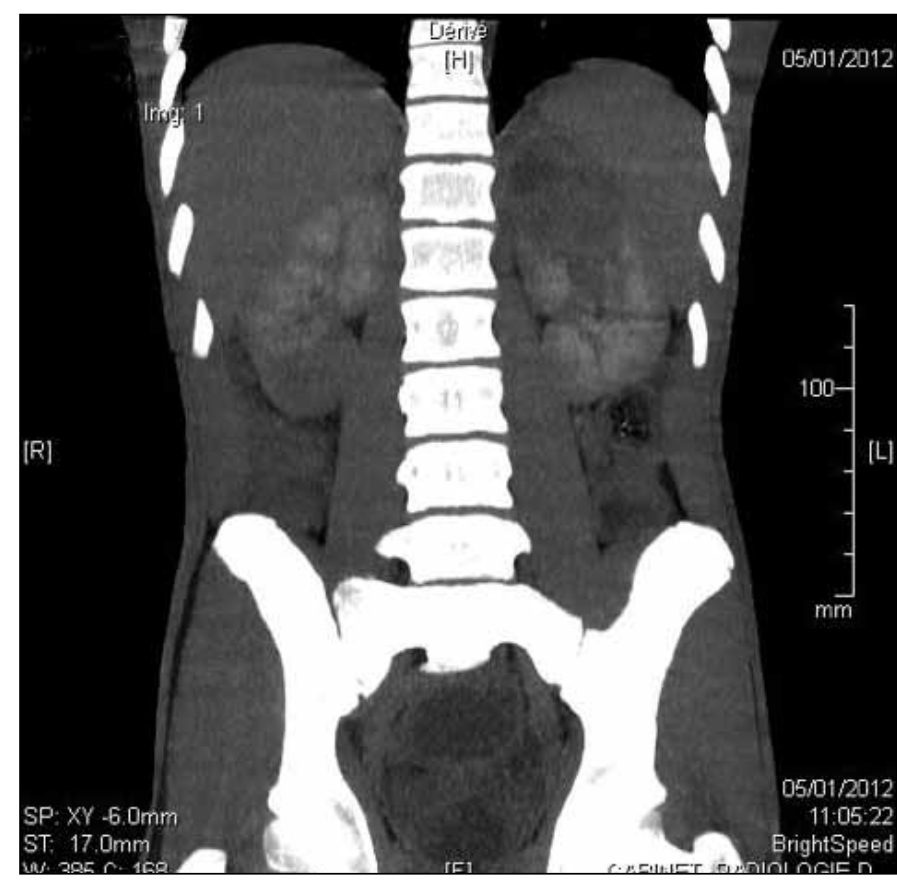

Fig. 1. Coupe frontale en en phase parenchymateuse à l'urotomodensitométrie.

tiocytes spumeux, de nombreux éléments lymphoplasmocytaires et de quelques cellules épithélioïdes éparses, le tout étant associé à une fibrose interstitielle diffuse avec atrophie tubulaire et aspect de " pain à cacheter » (figure 4, figure 5). Le diagnostic de PXG a été retenu. Le patient a reçu un traitement antibiotique à base de fluoroquinolone pendant un mois. L'évolution a été marquée par la résorption complète des lésions visibles à la tomodensitométrie après un mois et la disparition des douleurs lombaires. Le patient vit toujours après un an. Sa fonction rénale est normale, et aucune anomalie n'a été décelée à la tomodensitométrie de contrôle.

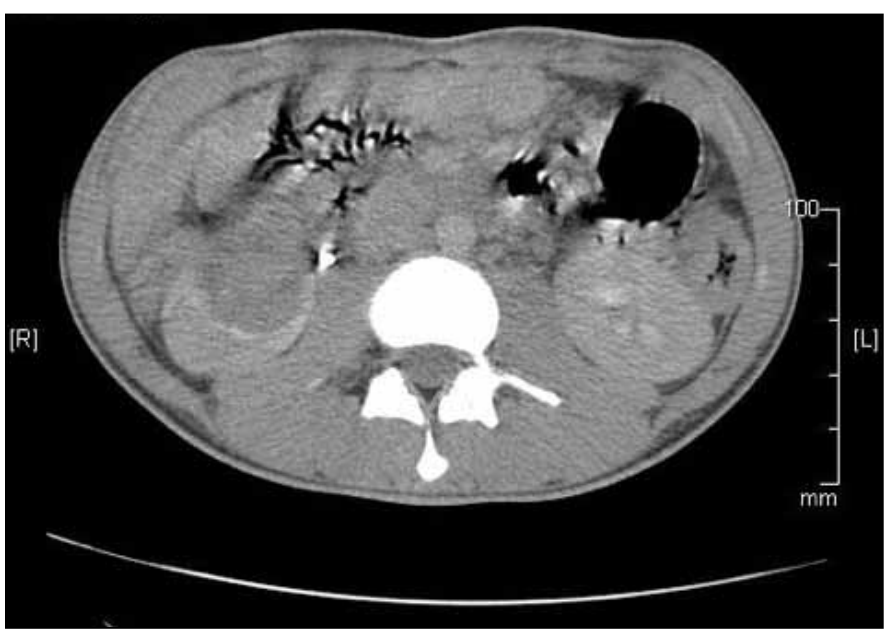

Fig. 3. Coupe axiale montrant la lésion polaire inférieure droite à l'urotomodensitométrie.

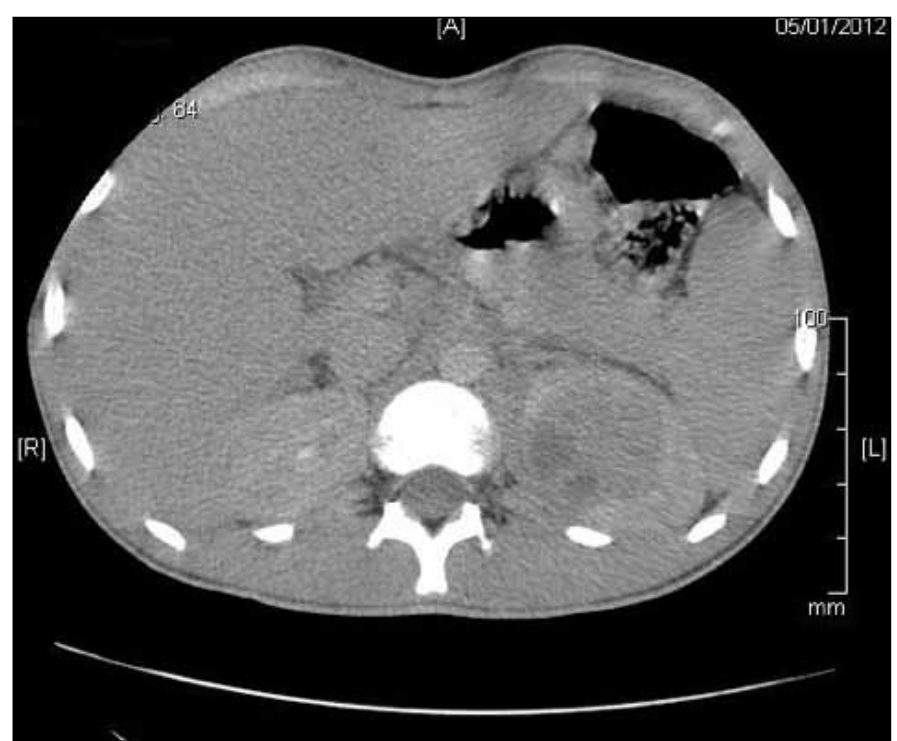

Fig. 2. Coupe horizontale montrant la lésion polaire supérieure gauche à l'urotomodensitométrie.

\section{Discussion}

La PXG bilatérale est extrêmement rare. On a signalé dans la littérature 10 cas de PXG diffuse bilatérale et 5 cas de PXG focale bilatérale au cours des 40 dernières années (voir le tableau 2$)^{4-18}$. Différents facteurs étiologiques ont été proposés : I'obstruction chronique due au calcul et l'infection urinaire sont les facteurs les plus reconnus ${ }^{19}$.

Selon les données colligées dans la littérature, la PXG peut occasionner des douleurs abdominales ou lombaires, de la fièvre et un amaigrissement. La présence $d^{\prime}$ 'une masse palpable est inconstante. Sur le plan biologique, la protéinurie, I'anémie, l'hyperleucocytose et l'insuffisance rénale sont d'autres symptômes associés. Dans le cas qui nous occupe, le patient présentait uniquement des lombalgies sans autre signe clinique ou biologique susceptible de conforter le diagnostic de PXG $^{19}$.

Les cinq cas de PXG focale bilatérale rapportés dans la littérature étaient tous apyrétiques.

Les résultats de l'urotomodensitométrie penchaient en faveur d'une tumeur rénale bilatérale. Étant donné la possibilité de lésions rénales tissulaires bilatérales, la biopsie rénale était indiquée. La culture cellulaire et l'examen bactériologique de la biopsie rénale ont permis d'identifier le germe en cause et de réaliser un antibiogramme ${ }^{20}$.

Cependant, un problème se posait : on ne peut éliminer I'hypothèse de tumeur rénale même en cas de biopsie néga-

Tableau 1. Mesure de la densité des lésions rénales en unités Hounsfield (UH)

\begin{tabular}{lccccc}
\hline Temps & Sans injection & Artériel & Portal & Tardif 1 & Tardif 2 \\
\hline Lésion droite & 34 & 42 & 54 & 56 & 50 \\
Lésion gauche & 31 & 40 & 50 & 48 & 44
\end{tabular}




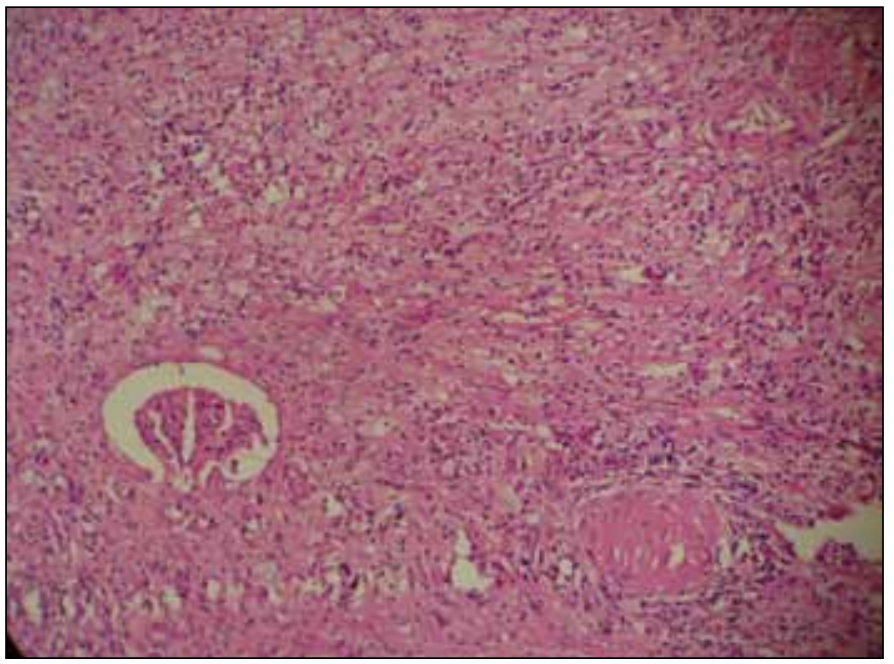

Fig. 4. Infiltrat inflammatoire polymorphe avec éléments histiocytaires à cytoplasme spumeux (hémalun-éosine $\times 10$ ).

tive. Une néphrectomie partielle polaire inférieure droite a donc été réalisée devant la forte suspicion de tumeur rénale. Ce n'est qu'à l'examen anatomopathologique de la pièce opératoire que le diagnostic de PXG a été retenu. On a pu opter pour une approche conservatrice, mais rappelons que le succès du traitement conservateur de la PXG focale dépend fondamentalement de l'absence d'obstruction urétérale sous-jacente.

\begin{tabular}{|c|c|c|c|c|}
\hline Cas & Fièvre & Forme & Néphrectomie & Évolution \\
\hline Rossi $1968^{4}$ & + & $\mathrm{D}$ & - & Décès \\
\hline Vandendris $1976^{5}$ & - & $\mathrm{F}$ & + partielle & Vivant \\
\hline Husain $1979^{6}$ & - & $\mathrm{D}$ & + bilatérale & Vivant \\
\hline Smith $1981^{7}$ & + & $\mathrm{D}$ & - & Vivant \\
\hline Braun 19858 & $\begin{array}{c}\text { Non } \\
\text { précisée }\end{array}$ & $\mathrm{D}$ & Non précisée & Décès \\
\hline Golomb $1986^{9}$ & + & D & + bilatérale & Décès \\
\hline Kural $1987^{10}$ & - & $\mathrm{D}$ & + bilatérale & Vivant \\
\hline Goswami $1988^{11}$ & + & $\mathrm{D}$ & - & Décès \\
\hline Bazeed $1989^{12}$ & $\begin{array}{c}\text { Non } \\
\text { précisée }\end{array}$ & $\mathrm{D}$ & Non précisée & Décès \\
\hline Akhtar $1992^{13}$ & + & $\mathrm{D}$ & + bilatérale & Vivant \\
\hline Perez $1993^{14}$ & - & $F$ & + partielle & Vivant \\
\hline Ozcan $1995^{15}$ & - & $\mathrm{F}$ & + gauche & $\begin{array}{c}\text { Non } \\
\text { précisée }\end{array}$ \\
\hline Suzer $1996^{16}$ & - & $F$ & + gauche & Vivant \\
\hline Karadeniz $2002^{17}$ & - & $F$ & +droite & Vivant \\
\hline Tsai $2008^{18}$ & - & $\mathrm{D}$ & - & Vivant \\
\hline Notre cas & - & $\mathrm{F}$ & $\begin{array}{c}\text { partielle } \\
\text { droite }\end{array}$ & Vivant \\
\hline
\end{tabular}

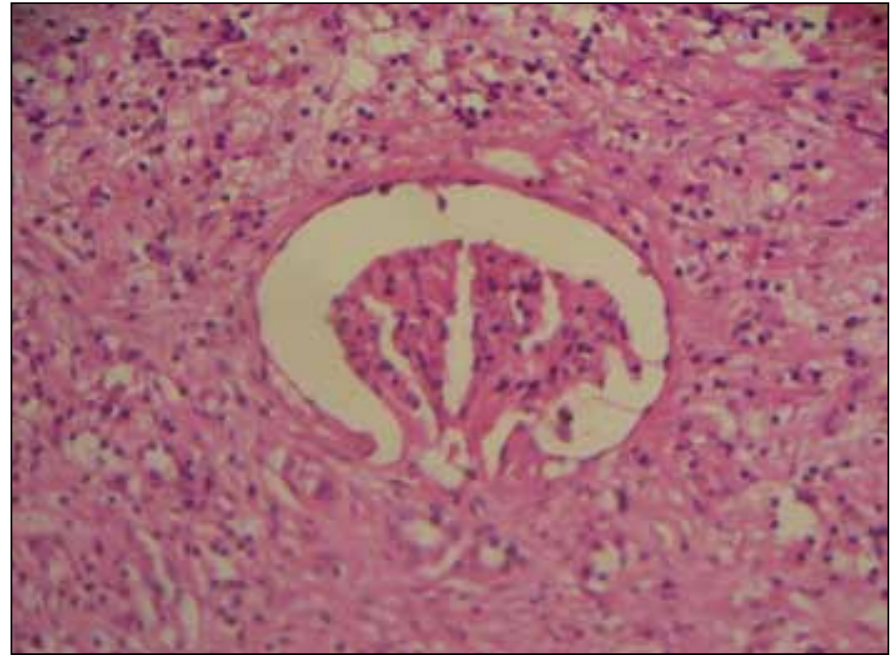

Fig. 5. Infiltrat inflammatoire avec aspect de " pain à cacheter " (hémalunéosine $\times 40$ ).

Les cinq cas de PXG focale bilatérale rapportés dans la littérature ont connu une évolution favorable à la suite $d^{\prime}$ une néphrectomie partielle bilatérale, dans deux cas, et d'une néphrectomie partielle unilatérale, dans trois cas. D'après I'analyse des cas de PXG bilatérale, il semble que le caractère diffus de la pyélonéphrite et la fièvre soient des facteurs de mauvais pronostic. La surveillance, pendant plusieurs années, des cas traités est nécessaire dans la mesure où des associations avec un carcinome à cellules rénales ont été signalées ${ }^{21}$.

\section{Conclusion}

La PXG bilatérale est une situation clinique délicate dont le diagnostic n'est pas toujours aisé. En présence de lésions bilatérales, il peut être indiqué de procéder à une biopsie rénale. Même si celle-ci s'avère négative, on ne peut éliminer l'hypothèse d'une tumeur rénale. Le pronostic semble meilleur en cas de PXG focale apyrétique.

Competing interests: Dr. El Yacoubi, Dr. Ziouziou, Dr. Zizi, Dr. Jahid, Dr. Karmouni, Dr. El Khader, Dr. Koutani and Dr. Andaloussi all declare no competing financial or personal interests.

This paper has been peer-reviewed.

\section{Références}

1. Korkes F, Favoretto RL, Bróglio M, et al. Xanthogranulomatous pyelonephritis: Clinical experience with 41 cases. http://dx.doi.org/10.1016/i.urology.2007.09.026

2. Tilkoff-Rubin NE, Cotran RS, Rubin RH. Urinary tract infection, pyelonephritis, and reflux nephropathy. Dans : Brenner BM (dir.). Brenner \& Rector's The Kidney, 7e édition. Philadelphia: Saunders; 2004:1554-5.

3. Parsons MA, Harris SC, Longstaff AJ, et al. Xanthogranulomatous pyelonephritis: A pathological, clinical and aetiological analysis of 87 cases. Diagn Histopathol 1983;6:203-19. 
4. Rossi P, Myers DH, Furey R, et al. Angiography in bilateral xanthogranulomatous pyelonephritis. Radiology 1968;90:320-1. http://dx.doi.org/10.1148/90.2.320

5. Vandendris M, Struyven J, Mathieu J, et al. Bilateral xanthogranulomatous pyelonephritis. J Radiol Electrol Med Nud 1976;57:891-3.

6. Husain I, Pingle A, Kazi T. Bilateral diffuse xanthogranulomatous pyelonephritis. Br J Urol 1979;51:162-3. http://dx.doi.org/10.1111/i.1464-410X.1979.tb02854.x

7. Smith FR. Bilateral xanthogranulomatous pyelonephritis. Br J Urol 1981;53:81. http://dx.doi. org/10.1111/j.1464-410X.1981.tb03134.x

8. Braun G, Moussali L, Balanzar JL. Xanthogranulomatous pyelonephritis in children. J Urol 1985;133:236-9.

9. Golomb J, Solomon A, Peer G, et al. Bilateral metachronous xanthogranulomatous pyelonephritis in endstage renal failure. Urol Radiol 1986;8:95-7. http://dx.doi.org/10.1007/BF02924086

10. Kural AR, Akaydin A, Oner A, et al. Xanthogranulomatous pyelonephritis in children and adults. $\mathrm{Br} J$ Urol 1987;59:383-5. http://dx.doi.org/10.1111/i.1464-410X.1987.tb04830.x

11. Goswami AK, Suryaprakash B, Malik AK, et al. Development of fatal bilateral xanthogranulomatous pyelonephritis in a paraplegic patient: case report. Paraplegia 1988;26:62-5. http://dx.doi.org/10.1038/ sc. 1988.14

12. Bazeed MA, Nabeeh A, Atwan N. Xanthogranulomatous pyelonephritis in bilateral patients: A report of 25 cases. J Urol 1989;141:261-4.

13. Akhtar M, Qunibi W. Bilateral xanthogranulomatous pyelonephritis involving native kidneys in a rena transplant recipient: Association with renal cell carcinoma and amyloidosis. Am J Kidney Dis 1992;20:28993. http://dx.doi.org/10.1016/S0272-6386(12)80704-9
14. Perez LM, Thrasher JB, Anderson EE. Successful management of bilateral xanthogranulomatous pyelonephritis by bilateral partial nephrectomy. J Urol 1993;149:100-2.

15. Ozcan H, Akyar S, Atasoy C. An unusual manifestation of xanthogranulomatous pyelonephritis: Bilateral focal solid renal masses. AJR Am J Roentgenol 1995;165:1552-3. http://dx.doi.org/10.2214/ air.165.6.7484615

16. Suzer 0 , Baltaci S, Kuzu I, et al. Bilateral xanthogranulomatous pyelonephritis in a child. Br I Urol 1996;78:950-1. http://dx.doi.org/10.1046/i.1464-410X.1996.131229.x

17. Karadeniz $C, 0$ guz $A$, Ataoglu 0 , et al. Primary renal lymphoma and xanthogranulomatous pyelonephritis in childhood. Nephrol 2002;15:597-600.

18. Tsai KH, Lai MY, Shen SH, et al. Bilateral xanthogranulomatous pyelonephritis. J Chin Med Assoc 2008;71:310-4. http://dx.doi.org/10.1016/S1726-4901(08)70128-X

19. Chuang CK, Lai MK, Chang PL, et al. Xanthogranulomatous pyelonephritis: Experience in 36 cases. $J$ Urol 1992; 147:333-6.

20. Reul 0, Waltregny D, Boverie J, et al. Pseudo-tumoral xanthogranulomatous pyelonephritis: Diagnosis by percutaneous biopsy and success of conservatory treatment. Prog Urol 2001;11:1274-6.

21. Huisman TK, Sand JP. Focal xanthogranulomatou pyelonephritis associated with transitional cell carcinoma. Urology 1992;39:281-4. http://dx.doi.org/10.1016/0090-4295(92)90307-I

Correspondence: Dr. Imad Ziouziou, Service d'urologie B, CHU lbn Sina, Faculté de médecine et pharmacie de Rabat, Université Mohamed V souissi, Rabat, Maroc; imadziouziou@hotmail.com 